\title{
Impacto de la longitud del introductor en procedimientos coronarios transradiales: Estudio clínico randomizado analítico.
}

\author{
Patricio Maragaño ${ }^{1}$, Claudio Pacheco ${ }^{1}$, Gonzalo Ramírez ${ }^{a}$, Cristina Cerro ${ }^{1, b}$ y Nicolás García ${ }^{a}$ \\ 1 Unidad Hemodinamia Hospital Regional de Talca \\ a Interno Medicina Universidad Católica del Maule \\ b Enfermera Unidad Hemodinamia Hospital Regional de Talca
}

Objetivos: El objetivo principal del estudio fue conocer el impacto de la longitud del introductor en la aparición de vasoespasmo radial en pacientes sometidos a cateterización coronaria. Los objetivos secundarios fueron conocer la relación entre la longitud y dolor al retirarlo, tiempo de procedimiento, radiación y analizar los factores de riesgo sugeridos para espasmo radial. Material y Métodos: Se realizó un estudio clínico randomizado analítico de 99 pacientes con indicación de cateterización coronaria. Se randomizaron a dos grupos y se les realizó el procedimiento con diferentes longitudes de introductor $(25 \mathrm{~cm}$ y $10 \mathrm{~cm})$, ambos con cubierta hidrofílica. Se registró el tiempo de procedimiento, dosis de radiación, radioscopia, dolor al retirar el introductor y vasoespasmo radial.

Resultados: En el total de pacientes el procedi- miento fue exitoso. Del total de pacientes, en 12 se observó espasmo radial, encontrándose una menor incidencia de vasoespasmo $(18,75 \%$ vs $5,88 \%, \mathrm{p}=0,005)$ y menor dolor al retirar el introductor $(43,75 \%$ vs $21,57 \%$, $\mathrm{p}=0,018)$ en pacientes con introductor corto. No se observaron diferencias entre ambos grupos en relación a los otros factores. Diabetes y género femenino se mostraron como predictores independientes de vasoespasmo radial en pacientes sometidos a procedimientos coronarios transradiales.

Conclusiones: El uso de un introductor corto disminuye la incidencia de vasoespasmo radial y dolor al retirar el introductor durante procedimientos coronarios transradiales.

Palabras claves: Angiografía coronaria, Angioplastía, Vaso espasmo. 


\section{Abstract: Impact of introducer length in trans-radial coronary interventions: a clinical randomized study}

Aim: Radial artery spasm producing severe pain is the most common complication of trans-radial approach for coronary artery catheterization. It remains unclear whether the length of the introducer is related to this complication. The aim of this study was to assess the impact of introducer length on radial artery spasm and, also, to identify variables associated to this complication.

Methods and results: 99 patients were randomized to receive either a long $(25 \mathrm{~cm})$ or a short $(10$ $\mathrm{cm}$ ) introducer for coronary catheterization. Both types of introducer were hydrophilic coated. The procedure was successful in all subjects. Radial artery spasm de- veloped in 12 patients, $18.8 \%$ of those receiving a long introducer vs $5.9 \%$ of those receiving the short one $(\mathrm{p}=0.005)$. Corresponding values for sheath retrieval pain were $21.6 \%$ and $43.8 \%$, respectively $(\mathrm{p}=0.018)$. No between-group difference was observed in radiation dose, radioscopy time and total procedure time. Diabetes and female gender were predictors of radial artery spasm.

Conclusion: Use of a shorter hydrophilic coated introducer resulted in a lesser incidence of radial artery spasm and associated pain, during coronary catheterization.

Key words: vasospasm, angiography, cardiac catheterization

\section{Introducción}

Desde que Campeau comenzó con la técnica de acceso transradial (ATR) para la realización del cateterismo cardíaco en 1989, cada vez está siendo adoptado por más cardiólogos intervencionistas ${ }^{1}$. Se ha documentado las ventajas de este acceso por sobre el acceso femoral en términos de ofrecer mayor seguridad y comodidad para el paciente al disminuir las complicaciones locales, menor hemorragia, eventos isquémicos y malestar con tasas similares de éxito en el procedimiento y deambulación precoz ${ }^{2,3}$. Sin embargo, la complicación más común del ATR es el espasmo de la arteria radial, el cual tiene una incidencia de un $14,7 \%$, causando un significativo malestar en el paciente y disminuyendo la tasa de éxito del procedimiento ${ }^{4}$.

Dentro de los factores de riesgo para desarrollar un espasmo radial no se considera el impacto que podría tener el largo del introductor. Existe sólo un estudio al respecto ${ }^{5}$, y la decisión del largo del introductor se basa en consideraciones empíricas

El objetivo principal del estudio fue conocer el impacto de la longitud del introductor en la aparición de espasmo arterial radial, así como en el tiempo de radioscopía, dosis de radiación y el tiempo total del procedimiento. Dentro de los objetivos secundarios se deseaba conocer la incidencia total de espasmo radial en nuestra población, la relación entre la longitud del introductor y el dolor al retirarlo y analizar los factores de riesgo actualmente sugeridos para espasmo radial.

\section{Material y Métodos}

Un total de 99 pacientes considerados para intervención de cateterismo cardíaco por vía transradial, entre septiembre de 2011 y marzo de 2012 fueron seleccionados para participar en el estudio, obteniendo el consentimiento informado de todos los pacientes previamente. Los criterios de exclusión fueron los siguientes: pacientes que requerían procedimiento de urgencia, hemodinámicamente inestables, con una fístula arteriovenosa en el antebrazo o que se negaran a participar.

Se realizó un estudio clínico prospectivo randomizado analítico, asignando a los pacientes aleatoriamente a dos grupos con diferentes longitudes de introductor, usando una tabla estándar de randomización. Los introductores podía ser de dos tipos: un introductor largo de $25 \mathrm{~cm}$ y un introductor corto de $10 \mathrm{~cm}$, ambos 
Pinnacle Terumo ${ }^{\circledR}$ (Terumo Interventional Systems, Somerset, New Jersey), con cubierta hidrofílica. Los introductores utilizados en este estudio (99) fueron donados por Terumo Interventional System, quienes no se involucraron en el diseño del estudio, recolección y análisis de los datos o en la redacción de este artículo.

El abordaje por vía radial es el acceso de elección en nuestro centro. $\mathrm{Al}$ ingreso al pabellón el brazo del paciente se ubicó en posición anatómica con la muñeca hiperextendida. Se aplicó anestesia local con lidocaína al 2\% después de preparar la piel del sitio de punción. La canulación arterial se realizó mediante técnica de Seldinger con una aguja de 21-gauge, por la cual se introdujo una guía de 0,118 pulgadas de nitinol con punta de platino. Se introdujo un introductor de $6 \mathrm{~F}$, de longitud 10 o $25 \mathrm{~cm}$ modelo Pinnacle Terumo ${ }^{\circledR}$, por el cual se administraron $5.000 \mathrm{U}$ de heparina. Se evitó el uso rutinario de un agente espasmolítico para disminuir el impacto que éste podría tener en el espasmo arterial. Esto es una práctica estándar en nuestra institución. Sólo se usaron agentes espasmolitícos (Verapamilo $5 \mathrm{mg}$ ) en los casos de espasmo radial. El resto del procedimiento se realizó acorde a la preferencia del operador. Se midió el tiempo total del procedimiento, desde la punción arterial hasta la última inyección. Junto a esto se registró la dosis de radiación y el tiempo de radioscopia total. El introductor fue retirado al finalizar el procedimiento y la hemostasia se logró en el laboratorio de cateterización a través de un mecanismo de compresión.

Criterios de espasmo radial

El espasmo de la arteria radial fue definido clínicamente por dolor percibido por el paciente o dificultad percibida por el operador durante la inserción, manipulación y retiro del introductor o el catéter.

Los operadores reportaron el espasmo en las siguientes condiciones: el paciente refirió dolor continuo en el antebrazo; el paciente refirió dolor en el antebrazo sólo durante la manipulación del catéter; el paciente reportó dolor en el antebrazo durante la inserción o retiro del introductor, y también cuando la rotación /manipulación del catéter fue difícil durante el procedimiento y cuando se notó resistencia aumentada durante el retiro del introductor. Se definió como espasmo arterial cuando se presentaron al menos dos de los criterios, o sólo uno si fue necesario administrar vasodilatadores intra-arteriales.

Los pacientes reportaron el dolor percibido al retirar el introductor bajo la siguiente puntuación: $1=\operatorname{Sin}$ dolor, 2 = Dolor leve, $3=$ Dolor moderado y $4=$ Dolor intenso.

Se consideró espasmo radial cuando el paciente reportó dolor moderado a intenso al retirar el introductor

\section{Análisis estadístico}

El análisis estadístico fue realizado mediante los programa SPSS 17.0 y STATA: Data Analysis and Stadistical Software. Las variables continuas fueron expresadas como media \pm desviación estándar y las categóricas en porcentajes. Tras dividir a la población en 2 grupos según la longitud del introductor, se comparó el valor de las variables mediante el test de Student para las continuas y la prueba de X2 para las categóricas, y se consideró significativo un valor $\mathrm{p}<0.05$. Se utilizó un modelo de regresión logística para las variables a estudiar como predictores de espasmo radial. Las variables incluidas en el modelo fueron la edad, género, IMC, diabetes, hiperlipidemia, HTA, tabaquismo y tiempo del procedimiento.

\section{Resultados}

La población estudiada compuesta por 99 pacientes, intervenidos entre Septiembre del 2011 y Marzo del 2012, presentó un promedio de edad de 61,5 años, de los cuales $68(68,7 \%)$ pacientes fueron hombres. En relación a factores de riesgo cardiovasculares, $67(67,7 \%)$ eran hipertensos, $16(16,2 \%)$ tenían dislipidemia, $17(17,2 \%)$ eran fumadores y $28(28,3 \%)$ eran diabéticos. En 51 pacientes, correspondiente al $51,5 \%$, se utilizó un introductor corto. El tiempo promedio desde la punción arterial hasta la última inyección de contraste fue de 11,43 \pm 7,15 minutos y la cantidad de radiación total fue de $2855 \pm 1381$ uGym$^{2}$. De toda la población estudiada, se reportó una incidencia de espasmo radial en $12(12.1 \%)$ de los pacientes.

Las características clínicas de la población incluida, distribuidas según la longitud del introductor radial se exponen en la Tabla 1. No hubo diferencias 
significativas entre ambos grupos.

En los $99(100 \%)$ pacientes, el procedimiento fue

\begin{tabular}{|c|c|c|c|}
\hline Variables & $\begin{array}{l}\text { Largo } \\
(\mathrm{n}=48)\end{array}$ & $\begin{array}{l}\text { Corto } \\
(\mathrm{n}=51)\end{array}$ & p Value \\
\hline $\begin{array}{l}\text { Edad (años) } \\
\text { IMC (kg/m2) } \\
\text { Sexo Masculino } \\
\text { Dislidemia } \\
\text { Hipertensión Arterial } \\
\text { Tabaquismo } \\
\text { Diabetes Melitus }\end{array}$ & $\begin{array}{l}59,73 \pm 11,70 \\
28,32 \pm 3,26 \\
32(66,7 \%) \\
8(16,7 \%) \\
29(60,4 \%) \\
8(16,7 \%) \\
12(25 \%)\end{array}$ & $\begin{array}{l}63,14 \pm 10,86 \\
27,44 \pm 3,29 \\
36(70,6 \%) \\
8(15,7 \%) \\
38(74,5 \%) \\
9(17,7 \%) \\
16(31,4 \%)\end{array}$ & $\begin{array}{l}0,068 \\
0,907 \\
0,674 \\
0,895 \\
0,134 \\
0,897 \\
0,482\end{array}$ \\
\hline
\end{tabular}

Valores: promedio \pm SD o n (\%), IMC: índice de masa corporal

exitoso. Sólo en 1 paciente se cambio a vía femoral por haberse generado un vasoespasmo severo.

Se observó la presencia de espasmo radial en 9 $(18,8 \%)$ pacientes en los que se utilizó un introductor largo y en $3(5,9 \%)$ pacientes en los que fue utilizado un introductor corto $(\mathrm{p}=0,05)$.

El dolor referido por el paciente al retirar el introductor (incluyendo todos los niveles de dolor evaluados), fue significativamente mayor en el grupo con introductor largo. Veintiún pacientes refirieron dolor, y de este grupo, $9(18,8 \%)$ en la categoría moderado-severo, en comparación con el grupo de introductor corto, que en 11 pacientes refirió dolor, de los cuales sólo 2 fueron de carácter moderado; el resto fue leve. La diferencia entre ambos grupos fue significativa $(\mathrm{p}=0.019)$.

No se observó diferencia significativa entre ambos grupos en relación a la cantidad de radiación total y el tiempo hasta la última inyección de contraste (Tabla 2).

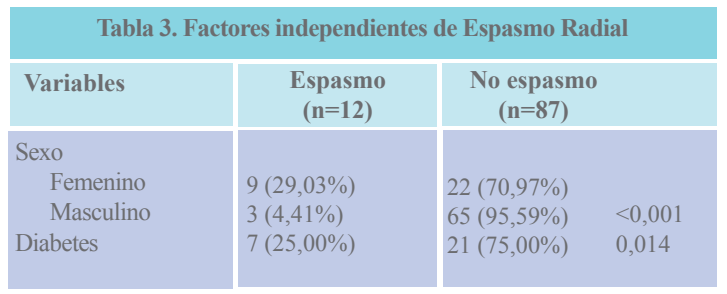

Valores: promedio $\pm S D$ o $n(\%)$

Como predictores de espasmo radial se identificó a la DM y al género femenino, no así la edad, IMC, HTA, dislipidemia ni tabaquismo (Tabla 3)

\begin{tabular}{|c|c|c|c|}
\hline Variables & $\begin{array}{l}\text { Largo } \\
(\mathrm{n}=48)\end{array}$ & $\begin{array}{l}\text { Corto } \\
(n=51)\end{array}$ & $\begin{array}{c}\text { p } \\
\text { Value }\end{array}$ \\
\hline Espasmo Radial & $9(18,75 \%)$ & $3(5,88 \%)$ & 0,050 \\
\hline Dolor al retiro & $21(43,75 \%)$ & $11(21,57 \%)$ & 0,018 \\
\hline $\begin{array}{l}\text { Tiempo } \\
\text { procedimiento ( } \mathrm{min})\end{array}$ & $10,79 \pm 5,04$ & $12,04 \pm 8,71$ & 0,196 \\
\hline $\begin{array}{l}\text { Dosis Radiación } \\
\text { (uGym2) }\end{array}$ & $2923,99 \pm 1414,45$ & $2815,43 \pm 1359,69$ & 0,646 \\
\hline Radioscopía & $4,69 \pm 3,56$ & $4,54 \pm 4,65$ & 0,571 \\
\hline
\end{tabular}

Valores: promedio \pm SD o $n(\%)$

\section{Discusión}

A pesar de las ventajas del ATR, éste tiene limitaciones, como una prolongada curva de entrenamiento, que resulta en una mayor exposición a radiación y el espasmo de la arteria radial. La arteria radial se caracteriza por ser un vaso con gran espesor de la pared debido a la gran cantidad de células musculares lisas dispuestas en capas concéntricas. Este factor anatómico, junto a la alta concentración de receptores alfa-1 genera que esta arteria tenga una mayor sensibilidad al espasmo comparada con otros vasos arteriales6. Existen pocos estudios que han analizado factores de riesgo implicados en el espasmo radial, reconociendo que el factor más importante podría ser una variación anatómica de la arteria radial $^{7}$, Sin embargo, la posible relación que podría tener el vasoespasmo con la longitud del introductor sólo ha sido estudiada en un estudio previo, el cual no encontró una relación significativa entre estas variables $^{5}$. La importancia de conocer los factores que se asocian al vasoespasmo radica en que junto a la falla en puncionar la arteria radial, son las principales causas de falla del procedimiento ${ }^{7}$. Sumado a esto, el vasoespasmo provoca un mayor dolor en el antebrazo los días siguientes a la intervención y el trauma físico endotelial que predispone a la formación de un trombo que posteriormente podría provocar la obstrucción de la arteria radial y, por consiguiente, problemas en un futuro procedimiento de cateterismo o en la necesidad de generar una fístula arteriovenosa para hemodiálisis. Sin embargo, una obstrucción persistente se observa sólo en el $6,9 \%$ de los pacientes ${ }^{\mathbf{5}, \mathbf{8}}$. Por estas razones, el objetivo principal de nuestro estudio fue analizar el impacto de la longitud del introductor en la incidencia de espasmo radial durante procedimientos coronarios transradiales. 
En nuestro estudio la tasa de vasoespasmo fue $12,1 \%$, valor que se asemeja al obtenido en otros estudios ${ }^{4}$. Sin embargo, una limitación común en los estudios de espasmo radial y posteriores conclusiones es la subjetividad de los criterios diagnósticos.

En el grupo de pacientes sometidos al procedimiento con introductor largo, 18,75\% desarrollaron espasmo radial comparado con sólo el 5,88\% del grupo de introductor corto que reportó vasoespasmo. Este resultado fue similar al observado en relación al dolor al retirar el introductor, reportándolo $43,75 \%$ de los pacientes del grupo largo comparado con $21,57 \%$ del grupo corto. Tanto la diferencia en la presencia de vasoespasmo como de dolor entre ambos grupos fue estadísticamente significativa. Este resultado difiere del encontrado por Rathore et al. Una posible explicación a esta diferencia es que en nuestro estudio sólo se utilizó introductores con cubierta hidrofílica, a diferencia del estudio de Rathore et al, en el cual se utilizó introductores con y sin cubierta hidrofílica.

Nuestro grupo también analizó predictores de espasmo radial en un procedimiento coronario transradial, actualmente señalados en la literatura. Se describe que la edad, género femenino IMC y diabetes mellitus serían factores que predicen el vasoespasmo. Específicamente se asocia a pacientes más jóvenes, de bajo IMC y en su mayoría mujeres ${ }^{5,8}$. En relación a estos factores, nuestra población que presentó espasmo radial fue en un $29 \%$ mujeres y un $25 \%$ diabéticos. Sólo estos factores fueron significativos en relación a predecir esta complicación, siendo un resultado concordante con estudios previos y posiblemente se deba a que anatómicamente, como ha sido reportado, las mujeres tienen un menor diámetro de la arteria radial, lo cual se ha señalado como un factor predictor de vasoespasmo radial ${ }^{\mathbf{9}}$.

La limitación más importante en nuestro estudio es el uso de criterios subjetivos para el diagnóstico de espasmo radial; sin embargo, para atenuar este factor se establecieron criterios diagnósticos en base a las distintas manifestaciones que podría tener el espasmo radial durante el procedimiento. En la literatura se ha encontrado una asociación entre las medidas objetivas y subjetivas para definir espasmo radial $^{10}$.

La segunda limitación fue que el estudio realizado fue ciego simple, lo que podría generar un sesgo de información en relación al diagnóstico de vasoespasmo; sin embargo, entre otros criterios, se utilizó el dolor al retirar el introductor, dato referido por el paciente.

\section{Conclusiones}

Este estudio randomizado permite concluir que el uso de introductor de corta longitud $(10 \mathrm{~cm})$ con cubierta hidrofílica se asocia a menor incidencia de espasmo de la arteria radial y a menor dolor durante el procedimiento. Ello se obtuvo sin un aumento del tiempo de procedimiento ni de la magnitud de la exposición a radiación. También hemos identificado que el género femenino y la DM son predictores independientes de espasmo arterial radial. En relación a la dislipidemia, hipertensión, tabaquismo y edad no se encontró una asociación estadística significativa con el espasmo radial.

Agradecimientos: Los autores agradecen a Carola Orrego por su excelente asistencia técnica. 


\section{Referencias:}

1 RAO S, COHEN M, KANDZARI D, BERTRAND OF, GILCHRIST IC. The transradial approach to percutaneous coronary intervention. J Am Coll Cardiol. 2010; 55: 2187-95.

2.- VOROBCSUK A, KONYI A, ARADI D, HORVATH IC, UNGI I, LOUVARD Y, et al. Transradial versus transferomal percutaneous coronary intervention in acute myocardial infarction: systematic overview and meta-analysis. Am Heart J. 2009; 158: 814-21.

3 JOLLY SS, AMLANI S, HAMON M, YUSUF S, PHIL M, Mehta S. Radial versus femoral access for coronary angiography or intervention and the impact on major bleeding and ischemic events: a systematic review and meta-analysis of randomized trials. Am Heart J. 2009; 157:132-40.

4 HILDICK-SMITH DJ, LOWE MD, WALSH JT, LUDMAN PF, STEPHENS NG, SCHOFIELD PM, et al. Coronary angiography from the radial artery-experience, complications and limitations. Intern J Cardiol. 1998; 64: 231-9.

5 RATHORE S, STABLES R, PAURIAH M, HAKEEM A, MILLS JD, PALMER ND, et al. Impact of length and hydrophilic coating of the introducer sheath on radial artery spasm during transradial coronary intervention. J Am Coll Cardiol Intv. 2010;
3:475-83

6 HE GW, YANG CQ. Comparison among arterial grafts and coronary artery. An attempt at functional classification. J Thorac Cardiovasc Surg. 1995; 109: 797-715.

7 KIEMENEIJ F, LAARMAN GJ, ODEKERKEN D, SLAGBOOM T, WEIKEN RVD. A randomized comparison of percutaneous transluminal coronary angioplasty by the radial, brachial and femoral approaches: the ACCESS study. J Am Coll Cardiol. 1997; 29:1269-75

8 RUIZ-SALMERÓN R, MORA R, VÉLEZ-GIMÓN M, ORTIZ J, FERNÁNDEZ C, VIDAL B, et al. Espasmo radial en el cateterismo cardíaco transradial. Análisis de los factores asociados con su aparición y de sus consecuencias tras el procedimiento. Rev Esp Cardiol. 2005; 58:504-11.

9 JIA DA, ZHOU YJ, SHI DM, LIU YY, WANG JL, LIU XL, et al. Incidence and predictors of radial artery spasm during transradial coronary angiography and intervention. Chin Med J. 2010; 123:843-47

10 KIEMENEIJ F, AIDER B, ECCLESHALL S, LAARMAN G, SLAGBOOM T, VAN DER WEIKEN R. Measurement of radial artery spasm using an automatic pull back device. Catheter Cardiovasc Interv. 2001; 54:437-41. 